\title{
Organización de la fuerza de ventas
}

\section{Organization of the sales force}

\author{
Sandra Luz Hernández Mendoza ${ }^{a}$, Danae Duana Avila ${ }^{b}$
}

\begin{abstract}
:
Nowadays, sales administration facilitates the processes of follow-up, generation, and business opportunities, in addition to keeping the consumers of products and services updated. Therefore, organizations must present integrated strategies to obtain better results; For this reason, sales management is a source of information for the marketing area since the focus of sales has been changed and strategies such as segmentation and the value proposition have been implemented to maintain lasting relationships with customers. which has given rise to the management of ARC customer relationships. Product development and innovation are fundamental to the survival of any organization; The company must respond in advance to customer requests by offering innovative products that meet their needs: if not, it will simply look to the competition to meet their wishes, which is why the importance of organizing the sales force through analysis and control of costs, promotions and publicity reflected within its organizational structure and type of organization.
\end{abstract}

Keywords:

Strategies, sales, customers, business.

\section{Resumen:}

Hoy en día la administración de ventas facilita los procesos de seguimiento, generación y oportunidades de negocio, además de que permite mantener actualizados a los consumidores de productos y servicios. Por lo anterior las organizaciones deben presentar estrategias integradas para obtener mejores resultados; por ello la administración de ventas es fuente de información para el área de mercadotecnia dado que se ha cambiado el enfoque que se tenían las ventas y se ha implementado estrategias como la segmentación y la propuesta de valor con el objetivo de mantener relaciones duraderas con los clientes, lo que ha dado origen a la administración de las relaciones con los clientes ARC. Desarrollo e innovación de productos son fundamentales en la subsistencia de cualquier organización; la empresa debe responder anticipadamente a las peticiones de los clientes ofreciendo productos innovadores que satisfagan las necesidades: de no ser así simplemente buscará en la competencia cumplir con sus deseos es por ello la importancia de la organización de la fuerza de ventas a través del análisis y control de costos, promociones y publicidad reflejada dentro de su estructura organizacional y tipo de organización.

Palabras Clave:

Estrategias, ventas, clientes, negocios.

a Profesor Profesor-Asignatura del Instituto de Ciencias Económico Administrativas. Universidad Autónoma del Estado de Hidalgo. Área Académica de Turismo-Campus ICEA-La Concepción, km 2.5, San Juan Tilcuatla Municipio de San Agustín Tlaxiaca Hidalgo. C.P. 42161. ORCID: https://orcid.org/ 0000-0002-2022-3135, e-mail: sandrahm@uaeh.edu.mx

b Profesor-Investigador del Instituto de Ciencias Económico Administrativas. Universidad Autónoma del Estado de Hidalgo. Área académica de Administración--Campus ICEA-La Concepción, km 2.5, San Juan Tilcuatla Municipio de San Agustín Tlaxiaca Hidalgo. C.P. 42161. ORCID: https://orcid.org/0000-0003-2286-2843,e-mail: duana@uaeh.edu.mx 


\section{Introducción}

La organización es una característica del proceso administrativo. En todo plan debe existir una división y asignación de las actividades para asegurar el mayor aprovechamiento de los recursos y el logro de metas y objetivos.

La organización de la fuerza de ventas está enfocada en ordenar las actividades del grupo de personas que conforman el área de ventas; además permite organizar a los vendedores, en función de los objetivos de la organización y de los recursos disponibles, las cuales pueden ser lineal, lineal y de staff, organización funcional e informal, así como por área geográfica, por producto, por funciones y por tipos de clientes [1].

Para una buena organización en la fuerza de ventas es importante analizar la estructura organizacional de la empresa, esta debe tener como propósito:

- Responder a las necesidades de los clientes

- Establecer las actividades de manera eficiente: división y especialización de las actividades

- Definir los canales de comunicación

- Estabilidad y continuidad del desempeño de la organización

- Coordinación e integración del área de ventas

De ahí que la estructura organizacional para el área de ventas esté diseñada en función de la organización, sus objetivos, estrategias y tareas; en función de los cambios que se den en la estrategia y objetivos que impactan directamente en el diseño de la estructura organizacional que se implementará [2].

Las clases organizacionales más comunes en el área de ventas son:

Organización lineal: es un modelo simple, utilizado frecuentemente por pequeñas organizaciones donde se encuentra el gerente de marketing que tiene a su cargo el gerente de ventas y pocos gerentes por regiones o cuentas, los cuales a su vez tienen subordinados.

Organización lineal y de staff: agrega más áreas funcionales y anexa asistentes de staff como apoyo al logro de las actividades que dan soporte al área de ventas, estás pueden ser: investigación de mercados, capacitación a la fuerza de ventas, pronósticos de ventas, servicios técnicos entre otros.

Organización funcional: está enfocada a la función; es decir, los niveles medios pueden controlar sus funciones y como consecuencia a quienes intervienen en ellas.

Organización informal: es la que realmente se sigue en la organización para hacer las cosas. Las organizaciones deben ser dinámicas y ajustarse a sí mismas y deberán encontrar la forma para concluir sus actividades con efectividad.

Muchas organizaciones, para organizar al área de ventas, suelen utilizar una estructura en relación con las áreas geográficas, los productos, los tipos de clientes y las funciones; es decir, se organiza a las ventas en el departamento de mercadotecnia según sean sus objetivos.

Organización por área geográfica: organización muy utilizada para tiendas de autoservicio, bancos, cadenas hoteleras, entre otras, donde precisamente se organizan por área geográfica. A los gerentes de ventas se les llama gerentes regionales (o distritales) de ventas. Este tipo de organización es útil cuando los clientes se encuentran dispersos, las variables regionales influyen en la conducta del consumidor y ésta difiere según el lugar.

Organización por producto: tiene como ventaja competitiva la constante innovación en los productos ofrecidos al mercado; los productos o líneas son lo suficientemente importantes e independientes que demandan trato especial.

Organización por funciones: se utiliza cuando las actividades son especializadas, no hay gran variedad de productos y las funciones son lo suficientemente importantes para asignar personal para su desarrollo. 
Retomando la importancia que tiene el cliente para toda la organización, en específico para ventas y mercadotecnia, en más de una ocasión es preciso definir la estructura organizacional en función de los clientes más rentables (o cuentas clave) para la organización [3].

Asignar las cuentas clave a altos ejecutivos de ventas: Existen organizaciones que utilizan como estrategia asignar a sus vendedores estrella, o de excelente desempeño y que están en el nivel jerárquico más alto, las cuentas clave, al ser éstas las que representan mayor utilidad para la organización, lo cual asegura que los clientes sean atendidos por personas experimentadas, responsables y comprometidas, capaces y con alto poder de decisión para ofrecer en forma acertada lo que busca el cliente [3].

\section{Dividir de manera independiente a las cuentas clave:}

Es importante que ventas y mercadotecnia dividan las cuentas clave, ya que mediante esta práctica se puede aplicar mercadotecnia que permita relaciones duraderas con los clientes más rentables [3].

Asignar una fuerza de ventas independiente para las cuentas clave: Muchas organizaciones asignan a sus vendedores más destacados cuentas clave, ya que aseguran mejor servicio, mejor trato y mejores ventas, pues se centran en pocos clientes [3].

\section{Referencias}

[1] Acosta, M. \& Salas L. (2018). La administración de ventas.3Ciencias Editorial Área de Innovación y Desarrollo S.L. Pág. 41-47 Recuperado de: https://www.3ciencias.com/wp-content/uploads/2018/02/La-administracion-de-ventas.pdf

[2] Aquino Morfin Belllanire. (2014). La importancia de las ventas en las empresas. Recuperado de: https://www.gestiopolis.com/la-importancia-de-las-ventasen-las-empresas/

[3] Fischer de la Vega, Laura Estela y Espejo Callado, Jorge (2011). Mercadotecnia, Cuarta Edición, Editorial Mc Graw-Hill, Pág. 40-47.

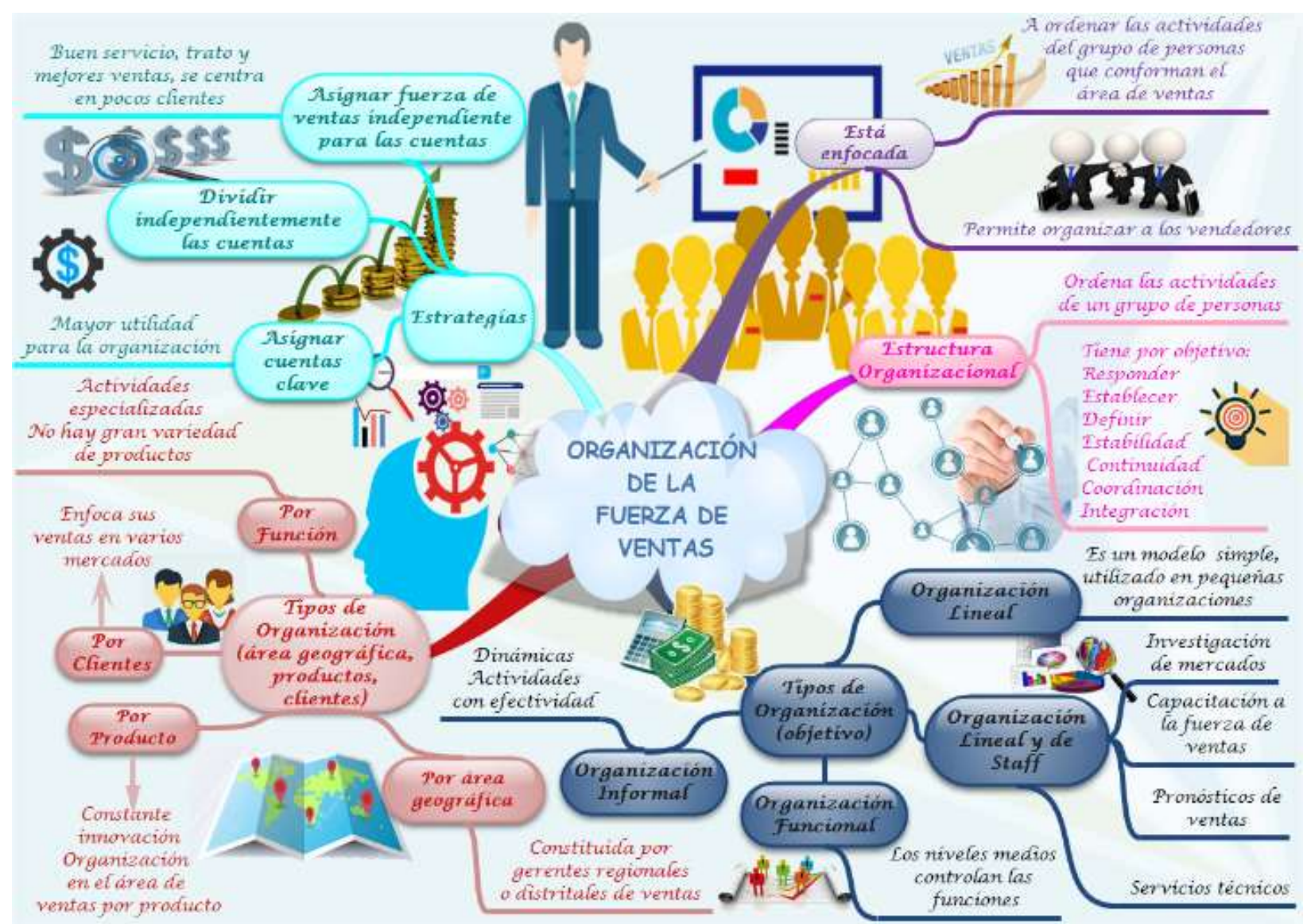

